\title{
The synthetic versatility of the Tiffeneau-Demjanov chemistry in homologation tactics
}

\author{
Stefan M. Kohlbacher ${ }^{1} \cdot$ Vivien-Sandra Ionasz $^{1} \cdot$ Laura Ielo $^{1} \cdot$ Vittorio Pace $^{1}[\mathbb{C}$
}

Received: 5 September 2019 / Accepted: 3 November 2019 / Published online: 20 November 2019

(c) The Author(s) 2019

\begin{abstract}
The Tiffeneau-Demjanov rearrangement can be regarded as an interesting alternative to the more common semi-pinacol transposition. It is usually employed for ring extension but, under specific conditions, it can also be used for ring contraction. Compared to other techniques, such as the Demjanov rearrangement or homologations with diazo compounds, the Tiffeneau-Demjanov pathway presents attractive features including high yielding and selective processes. Ring enlargements follow very strict and simple rules, such as the movement of the less substituted carbon and retention of the configuration. The rearrangement process is mainly affected by steric factors, due to presence of neighbouring groups, rather than electronic ones. The ring contraction may be achieved positioning the amine within the ring, thus achieving a high level of control. Unfortunately, applications of the reaction in modern homologation chemistry are rare; therefore, the aim of the review is re-proposing to the synthetic community the versatility of this venerable reaction and thus, spurring its employment for tackling challenging homologations processes.
\end{abstract}

\section{Graphic abstract}

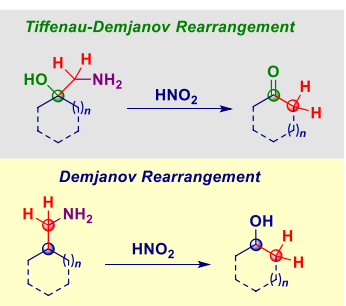

Keywords Tiffeneau-Demjanov $\cdot$ Rearrangement $\cdot$ Ring extension

\section{Introduction}

\section{Homologation chemistry: an overview}

Stefan M. Kohlbacher and Vivien-Sandra Ionasz contributed equally to this work.

Laura Ielo

ielo41@univie.ac.at

Vittorio Pace

vittorio.pace@univie.ac.at

1 Department of Pharmaceutical Chemistry, University of Vienna, Vienna, Austria
A carbon-chain homologation reaction can be defined as the elongation of the carbon skeleton chain with a methylene group which is inserted into the molecule to obtain the higher homolog [1]. This kind of reaction, in which one group is inserted, may also be applied to organometallic [2-21] and radical chemistry; however, the carbon-chain elongation is the most common one $[18,22]$. One of the simplest examples of a homologation pathway is the reaction of methanol with a mixture of carbon monoxide and hydrogen gases, also known as "synthesis gas", where a methylene 
Scheme 1

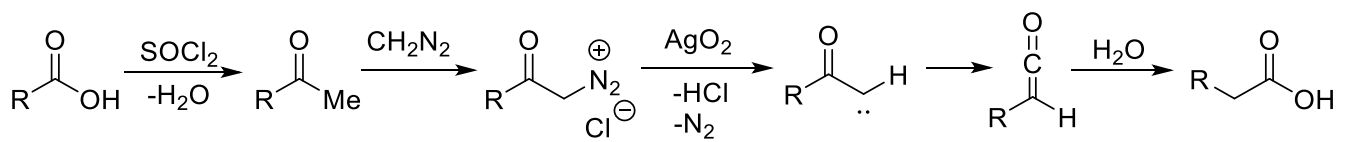

Scheme 2

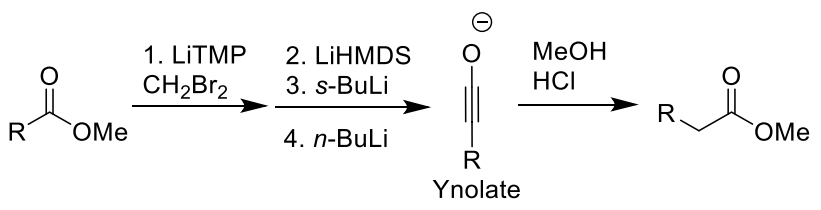

group is inserted between the carbon and the hydroxyl group leading to ethanol and water [23]. Homologation reactions are an essential tool in organic and medicinal chemistry considering the ever-increasing needing of more complex compounds and pharmaceuticals. However, this kind of reaction is not limited to acyclic carbon chains and it is very common in ring-expansion processes [24]. The homologation pathway is at its most useful when the synthesis of the lower homolog is much easier than that one of the required compound $[25,26]$.

One of the most popular homologation reactions is the addition of diazo compounds, such as the Arndt-Eistert reaction (Scheme 1) in which diazomethane is used as homologating agent providing $\mathrm{N}_{2}$ as excellent leaving group and making the reaction very clean and efficient [27]. This reaction is applied to extend carboxylic acids (1) into their higher homolog, by inserting a methylene unit. Using the halide of the carboxylic acid and an excess of diazomethane, the diazoketone (2) is produced in a nucleophilic substitution of the chlorine with diazomethane. Subsequently, the diazoketone is treated with water in the presence of a catalyst, such as silver, platinum or copper and, whenever necessary, applying heating and high pressure. Hereby, $\mathrm{N}_{2}$ is eliminated from the molecule leading to a ketene (3) after the rearrangement. Afterwards, the carbonyl carbon is attacked by a nucleophile, water, which leads the higher homolog of the carboxylic acid (4) [24]. The reaction may also be carried out with an alcohol, ammonia, or primary and secondary amines, leading to the corresponding ester or amides, respectively. During the reaction, the nitrogen is eliminated almost immediately followed by the rearrangement. Since the diazomethane is still active in the reaction, it can lead to undesired products by further homologation processes. Moreover, diazo compounds are explosive, very toxic, and difficult to manage. However, different procedures have been developed to produce diazomethane and its derivatives in situ [27].

Another very common direct homologation of esters is the Kowalski ester homologation (Scheme 2), useful for direct homologation of esters. It can be considered as
Scheme 3

(a)

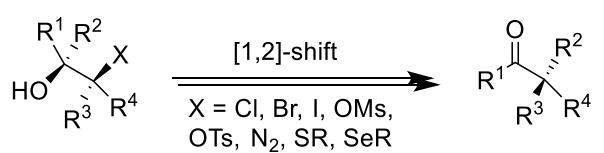

(b)

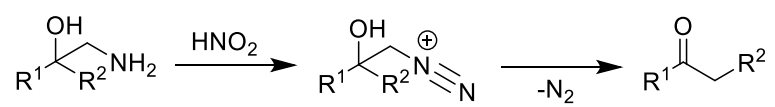

a safer alternative to the Arndt-Eistert synthesis, since it does not require the use of diazomethane [28]. This reaction can also be employed to synthesize $\beta$-amino esters, which are often present in drug molecules, starting from $\alpha$-amino esters with enantio- and diastereocontrol. During the Kowalski ester homologation an ynolate ion is produced as key intermediate, making the activation with diazomethane unnecessary and the reaction more attractive for larger scale approaches [26]. The reaction is carried out starting from an ester in presence of the methylenedibromide and a strong base at very low temperature and afterwards the ynolate ion formed is treated with an alcohol. Nevertheless, this one-pot approach produces very low yields and it is not applicable in large scale, therefore it was modified into a two-step reaction [25].

\section{The Tiffeneau-Demjanov reaction}

Rearrangement processes represent a straightforward alternative to homologation reactions with diazo compounds. A well-known rearrangement pathway is the semi-pinacol rearrangement [29] (Scheme 3a), performed on hydroxyl derivatives containing a good leaving group such as $\mathrm{Cl}, \mathrm{Br}$, I, or OMs in vicinal position. The elimination of these moieties is immediately followed by a [1,2]-shift, hydride or alkyl, of the neighbouring residues. A very interesting variant of the semi-pinacol rearrangement is the Tiffeneau-Demjanov (TD) rearrangement (Scheme $3 \mathrm{~b}$ ). It features a $\beta$-amino alcohol (5), which is then transformed into a diazide to be eliminated in the following rearrangement.

The TD rearrangement was discovered in 1937 by Bianka Tchoubar [30]. She realized that in the presence of an amino group, as a leaving group, in the semi-pinacols this rearrangement can be utilized for ring expansion after transforming the amine into a diazonium group. In 1949 she published three consecutive works on the importance of this transposition for 


\section{Scheme 4}

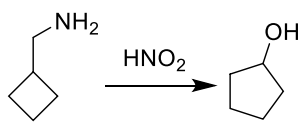

ring enlargement in synthesis, providing useful insights on mechanistic explanation.

Closely related, not only because of the name analogy, but also mechanistically, is the Demjanov rearrangement (Scheme 4).

The Demjanov rearrangement is a powerful method for ring enlargement of primary amines under acidic conditions. It can be applied to prepare different cyclic compounds but it works mainly for the preparation of five-to-seven-membered rings. Although these two reactions are very similar, they present differences in some aspects. One very important characteristic is the rearrangement product. A hydroxyl group is obtained in the Demjanov rearrangement, whereas the result of the TD rearrangement is a ketone, which eliminates an oxidation step after ring enlargement making it very convenient. Another critical aspect is the applicability of these reactions. Even though the Demjanov rearrangement is mainly reliable for five-to-seven-membered rings, the TD rearrangement has a wider application range and works perfectly also for rings of four-to-eight members [31]. Since homologation and rearrangement processes are extremely popular in drug synthesis, the selectivity is a crucial factor in the choice of the reaction to apply. Considering the yield, TD is clearly superior to the Demjanov rearrangement, which often leads to undesired side reactions with the solvent or in some cases can even lead to unspecific ring contractions. To date, stereoselective reactions are of high demand in synthesis, increasing the yield of one of the isomers and also avoiding a purification step afterwards. Since the initial molecular arrangement is rarely altered by the mild conditions of the reaction, the TD rearrangement provides an excellent method in the synthesis of highly modified compounds [32].

\section{Use of Tiffeneau-Demjanov reaction in ring enlargement}

As anticipated, the TD rearrangement can be considered as a variant of the pinacol rearrangement in which one hydroxyl group is exchanged against an amine, leading to the characteristic 2-amino-alcohol motif. The presence of an amine group distinguishes it from the conventional pinacol rearrangement and the hydroxyl group discriminates this reaction from the similar Demjanov rearrangement. The TD rearrangement consists of three steps (Scheme 5): (1) generation of a diazotization agent $\left(\mathrm{N}_{2} \mathrm{O}_{3}\right)$; (2) transformation of the amine group into a diazonium salt; (3) rearrangement of the residues from a carbonyl carbon to an amine carbon. The treatment of the
Scheme 5

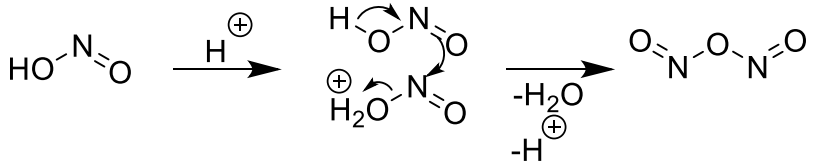

Step 2: Formation of diazonium ion

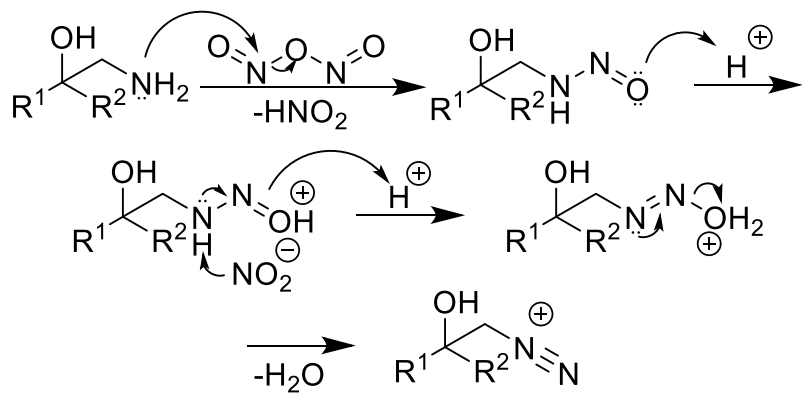

Step 3: Homologation

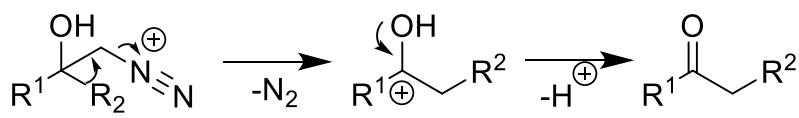

2-amino-alcohol with a nitrosonium ion generates a diazonium group under acidic conditions. This diazonium residue is an excellent leaving group and the reagent is able to undergo a rearrangement on the carbonyl carbon under heating conditions, eliminating $\mathrm{N}_{2}$ in the process [33].

The driving force behind this rearrangement is the stabilization of the obtained carbocation. After the elimination of nitrogen, a primary carbocation is obtained, which is then stabilized as a tertiary carbocation after the rearrangement. To undergo rearrangement, the moving residue needs to be in antiperiplanar orientation with respect to the eliminated nitrogen [23] (Scheme 6).

The moving carbon is usually that one present in the less substituted site of the hydroxyl group, but the movement of the more substituted carbon is also reported [34] (Scheme 7). The rearrangement proceeds under steric control, retaining the conformation of the moved carbon [35].

After rearrangement of the moved carbon, a proton is eliminated yielding a ketone in the process. In contrast to rearrangements induced by diazo compounds, the formed ketone is no longer reactive in the given setting, prohibiting the product 
Scheme 7<smiles>[R]C([C](C)C)[C](O)CCC(C)C</smiles><smiles>[R]C(C[C](C)C)C[C@H](O)CC(C)C</smiles>

from further rearrangements. This is due to the fact that the ketone was already formed in the second step of the TD reaction, consuming the entire diazotization agent in the solution. Only after the second step is completed, the solution is put under basic conditions to proceed with the rearrangement (Scheme 8).

The Demjanov rearrangement may undergo a [1,2]-hydride shift (Scheme 9), which is not possible for the carbonyl carbon in TD, proving to be superior compared to the Demjanov rearrangement. Additionally, the resulting carbocation is a carbonyl carbon for the TD rearrangement, stabilizing the positive charge much better than the secondary carbocation in the Demjanov rearrangement [36] (Scheme 10).

Therefore, in the TD rearrangement the ring expansion is much more favoured than the formation of side products, such as alcohols, compared to the Demjanov rearrangement. Additionally, the Demjanov rearrangement may eliminate $\mathrm{H}^{+}$ during the process, which can lead to the formation of various olefins. However, this is not possible for the TD rearrangement due to the quaternary nature of the carbonyl carbon [36].

\section{Steric aspects of the Tiffeneau-Demjanov reaction}

\section{Cyclic compounds}

The TD rearrangement behaves slightly different when applied to cyclic compounds, the most common
Scheme 9

(a)

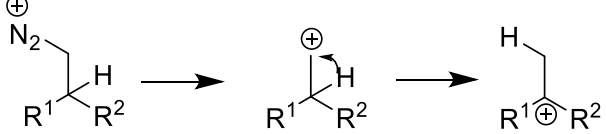

(b)<smiles>[R]CC([R1])[O-]</smiles>

Tiffeneau-Demjanov intermediate
Demjanov intermediate

Scheme 10

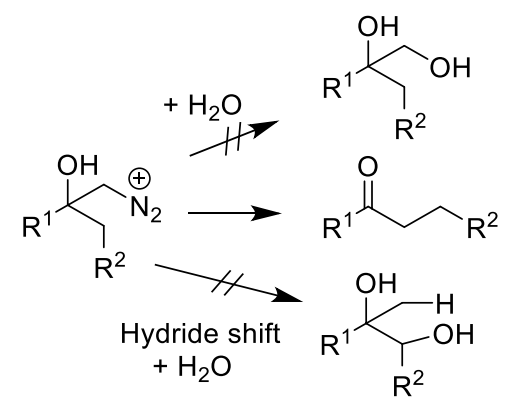

\section{Scheme 11}<smiles>[R]CCC([R])=O</smiles>

application, or acyclic compounds. In general, we can assert that the moving carbon is always the one present at the less substituted site of the starting ketone or hydroxyl group, especially in cyclic compounds [29]. This rule is mostly valid for substituents in position 2 .

The rearrangement can be applied to various complex ring systems and proceeds easily for most of them, even for bridged or polycyclic compounds, resulting in a cyclic

Scheme 8

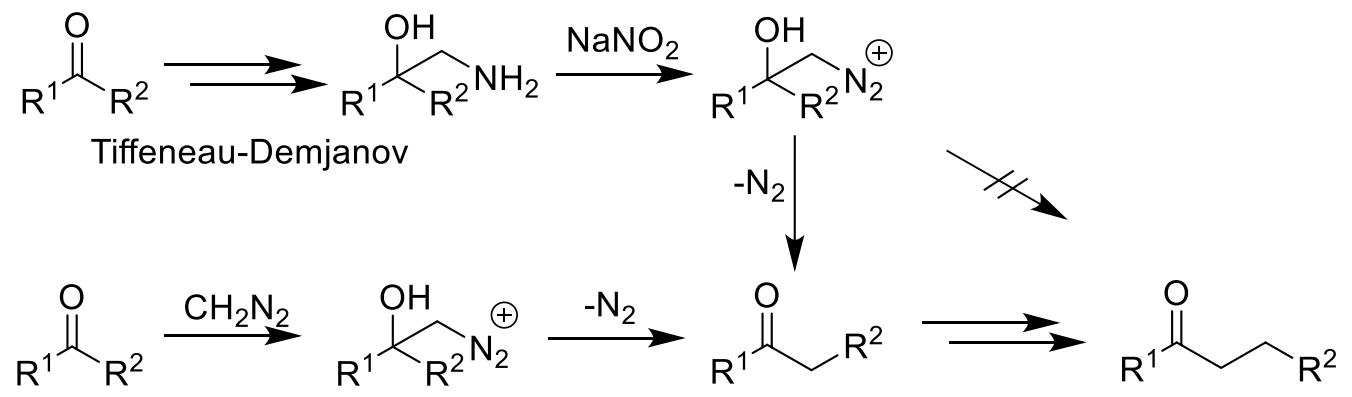

Diazo compounds 
ketone enlarged by a methylene group. The stereochemistry of the reaction is also valid for polycyclic compounds, such as steroids, which was investigated by Goldberg et al. [37]. During the steroids D-ring enlargement, the steric centre of the expanded ring kept its configuration and also, the less substituted carbon moved as expected. This can be confirmed by the application of the TD rearrangement to transisomer of these steroids, where the dominating product is the 17-keto-isomer, allowing the synthesis of these natural compounds in a highly selective way.

The moving carbon can also be controlled by hybridization state, $s p^{2}$ is preferred to $s p^{3}$ of the neighbouring carbon. Parham et al. [38] compared the aryl migration with the alkyl one. In a polycyclic system, the migration of the aryl group was favoured in comparison to the migration of the alkyl group by a factor of approximately 2 . This is attributed to the better stabilization of the carbocation of the phenyl ring instead of the alkyl ring and stands in contrast to the hypothesis that the less substituted carbon is migrating. Applying this rearrangement in fused ring systems, the favoured migration of the aryl group needs to be taken into account.

To date, it was always assumed that the hydroxyl and the amine groups are neighbouring each other. But what can happen if there is an additional carbon (a methylene group in the simplest case) inserted between these two groups? In this specific case, no ring enlargement has been observed but the deamination coupled with a [1,3]-hydride shift or the reaction with the solvent leading to a 1,3-diol [36] (Scheme 11).

\section{Acyclic compounds}

Even though the TD rearrangement is mostly used for ring enlargement, it can also be applied for the chain extension of acyclic compounds but in this case the stereochemistry is more complicated. The TD rearrangement only retains the configuration on the moving carbon, so the configuration on the alkyl carbon is not determined beforehand and it may change during the rearrangement.

In polycyclic systems the aryl migration was preferred to the alkyl migration due to superior stabilization of the carbocation of the phenyl ring (Scheme 12). This effect is even more pronounced in acyclic compounds, where the dominance of a migrating phenyl ring over an alkyl ring is expressed by a factor of around 30, compared to cyclic compounds with a factor of 2 . Since the phenyl ring is not sterically hindered anymore, as in the case of fused ring systems, it can occupy the preferred antiperiplanar orientation for migration, in which the $\pi$-orbitals may overlap in preferred orientation with the $\pi$-orbitals of the carbocation, which can be stabilized more efficiently [38].

Considering fused ring systems, the configuration on the non-migrating carbon atom might change. For acyclic
Scheme 12

(a)

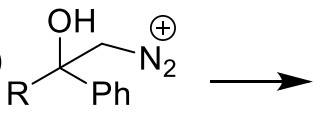<smiles></smiles><smiles>CCCCCC1(O)CCc2ccccc21</smiles><smiles>O=C1CCCc2ccccc21</smiles><smiles>O=C1CCc2ccccc2C1</smiles>

$1: 0.2-0.9$

compounds this effect can be even more pronounced due to their higher flexibility and it was investigated by Benjamin et al. [39, 40]. They considered differently substituted propanol derivatives to determine the exact mechanism of the TD rearrangement, but also the effects of various bulky groups on the configuration of the amino carbon. They realized that one crucial factor is that the deamination is not influenced by neighbouring groups, which allows a wide application of this rearrangement. They also found that the rearrangement process is mostly controlled by steric factors and that the electronic features can be neglected allowing simple stereo chemical control on the amino carbon by varying the substituent size. The resulting configuration can be determined by observing the transition state of the reacting compounds. For trans intermediates the configuration of the amino carbon is retained, whereas cis intermediates lead to a higher fraction of isomers with inverse configuration, since the transition state requires an eclipsed state of the bulky residues. Furthermore, they found out that complete deamination occurs within the reaction, leading to an open carbonium ion and no formed intermediates. This reinforces the assumption that the reaction is independent of electronic factors.

\section{Beta effect of silicon}

Silicon is known for its $\beta$-effect and consequently its stabilizing features on positively charged intermediates, directing the outcome of the reaction to go through intermediates with a positive charge in the $\beta$-position of the silicon [41]. Since the TD rearrangement proceeds under these exact circumstances, featuring a positively charged carbon in the transition state, it can be imagined that silicon, or other elements of the IV group, can be deployed to control which carbon will migrate during the rearrangement. Schow et al. [42] demonstrated that silicon and tin can be used to direct the outcome of the reaction, although the directing effect was not as strongly pronounced as expected (Scheme 13). 
By inserting silicon and tin substituents in the $\beta$-position of the carbonyl carbon, they expected to achieve control of the migrating carbon, that one close to the metal.

The weak directing effect can be asserted to the excellent leaving features of the diazonium group. As mentioned before, the reaction features an open carbenium ion without involvement of the migrating carbon in the transition state. Therefore, very little positive charge will be developed on the migrating carbon justifying the weak directing effect of the metal substituents. Furthermore, the developing bond is weakly polarised compared to the strong polarisation utilizing silicon or tin. Therefore, the choice of the different elements of the IV group can be applied to control the migrating site of the initial ketone, but the effects may be rather weak and other factors, such as steric clashes, may dominate. However, if various factors can be combined with the directing abilities of silicon, the resulting products can be pushed profoundly to one isomer.

\section{Improvement of the Tiffeneau-Demjanov reaction}

In the last few years, various improvements were made on the TD rearrangement, for example allowing a one-step reaction instead of three steps. This can be achieved using LTMSD (lithium trimethylsilyldiazomethane) (Scheme 14), which is a stronger nucleophile and a better reagent than diazomethane. LTMSD is first added to the ketone and only after all the reagents are consumed, the reaction will proceed under acidic conditions in a TD-like way, ensuring only single methylene homologation and a high product yield.

The ring expansion competes with the formation of an epoxide in this reaction, but under strong acidic conditions, the ring expansion will dominate and hardly any epoxide will be formed. Ring expansion occurs readily for ring sizes ranging from 4 to 7 members. Nearly all the expansions utilizing LTMSD behaved in the expected ways with the preference of aryl migration instead of the alkyl one and the less substituted carbon is favoured compared to a more

\section{Scheme 13}

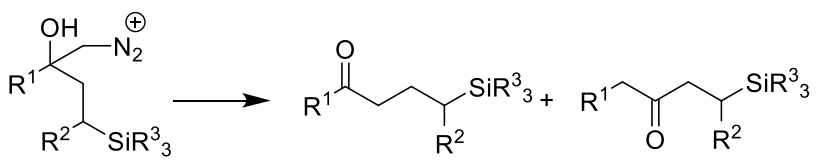

\section{Scheme 15}

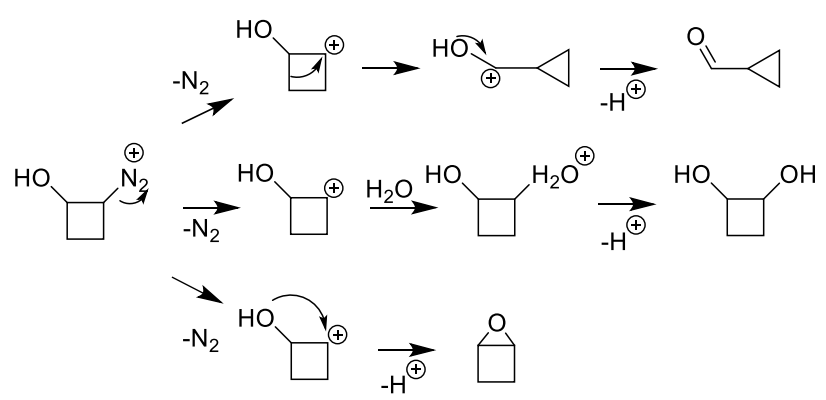

substituted one. An unexpected migration was observed with a silyl substituent. It was expected to have strong influence on the migration site due to its $\beta$-effect. However, considering the effects discussed above, in the silicon, possessing less impact on the stabilization of carbocations than in other reactions such as the Bayer-Villiger reaction, the preferred migration of the non-substituted site is not as surprising as one might think [43].

\section{Ring contraction and other possible side reactions}

Ring expansion easily proceeds if the amino group is not part of the ring. However, if the amino group is directly linked to the ring, the ring expansion is not possible anymore, since the carbocation will be formed inside the ring. Under these circumstances, the ring can undergo to other, often undesired, reactions such as ring contraction, formation of an epoxide, shifting of other substituents or the carbocation might even react with the solvent, similar to the Demjanov reaction [37, 44] (Scheme 15).

Steroids were a heavily investigated topic in the $1950 \mathrm{~s}$ and the TD rearrangement provided an excellent way to enlarge their rings as described before. In all of these cases the amino group was not directly connected to them. Due to the limited flexibility of these fused ring systems, the outcome of the rearrangement depends heavily on their configuration instead of the elimination of the nitrosonium ion. For instance, when the amino group is in axial orientation with respect to the steroid alkyl ring, either an epoxide can be formed or, if the neighbouring alcohol is tertiary, an [1,2]-alkyl shift takes places; however, the shifted alkyl group is not part of the ring system and therefore this rearrangement has no impact on the ring size. In contrast, if the

Scheme 14

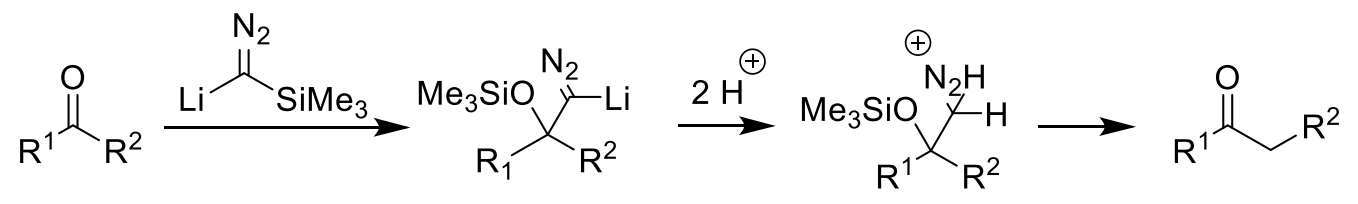


amino group is equatorially orientated, the elimination of the diazonium ion can lead to ring contraction or an epoxide formation, the dominating product. In both situations, the epoxide is only formed if the hydroxyl group is oriented in the other direction, equatorial or axial, respectively, and the configuration on the carbon atoms is retained [45]. During ring contraction, the nature of the substituent present in the same carbon of the amino group is not so important; however, it is reported that the presence of an aryl group inhibits the rearrangement, according to the stabilizing capabilities of the phenyl reported before [46].

\section{Application and use cases}

In the frame of the synthesis of the natural product (-)-isosteviol, Lohoelter et al. [47] individuated a series of important structural aspects such as its rigid nature and concave arrangement of the functional groups. Additionally, the presence of a substituent pointing into the cavity of the supramolecular architecture is essential for the transfer of chirality. Ring D was expanded through a TD-type ring enlargement. However, it did not form the desired structure with the keto function at position 22, but that one at position 21 (Scheme 16). When the scaffold was altered and an epoxide function was introduced before rearrangement under TD conditions, the correct structure was produced. This experiment did not only help to extend the use of an easy available and cheap resource for synthetic chemistry but also elucidated that the steric demand, which is caused by the unusual regioselectivity, is superior to the electronic arguments. Here, it could be observed that the TD rearrangement is very specific and always leads to the expected outcome, which in this case, meant that another function needed to be introduced into the molecule before to produce the expected compound [47].

Shi et al. [48] reported the application of this reaction for the total synthesis of two natural products, $( \pm)$-anislactone A and $( \pm$ )-merrilactone A (Scheme 17). These two compounds are of great interest due to their potent neurotrophic activity, which could lead to bioavailable therapeutics against neurodegenerative diseases. They were first isolated in 1989 [49] and 2000 [50], respectively, from plants of the Illicium genus. While four total synthesis approaches [33-36] have been already conducted for merrilactone A, only parts of the anislactone A synthetic route have been explored [55]. The goal of the study of Shi et al. was to produce both molecules in a total synthesis using the same carbon skeleton. A TD type reaction was successfully employed to enlarge the ring to a cyclopentanone by ethyl diazoacetate in the presence of $\mathrm{BF}_{3} \cdot \mathrm{Et}_{2} \mathrm{O}$, yielding $88 \%$ product in this step. The seminal work of Shi et al. is the first synthesis of anislactone A, which was achieved in 22 steps with an overall yield of 5.7\% through a common route for natural compounds. During this synthesis steps the stereoselectivity was of great importance. By selecting the TD rearrangement it was possible to homologate the whole molecule without changing [48].

Macías et al. [56] reported that the TD rearrangement leads to a different mechanism when performed in the dark. The homologation was observed to take place at the most hindered side of the carbonyl group, which is contrary to the literature for reactions under light, where the insertion takes place at the less hindered side. These findings were

\section{Scheme 16}

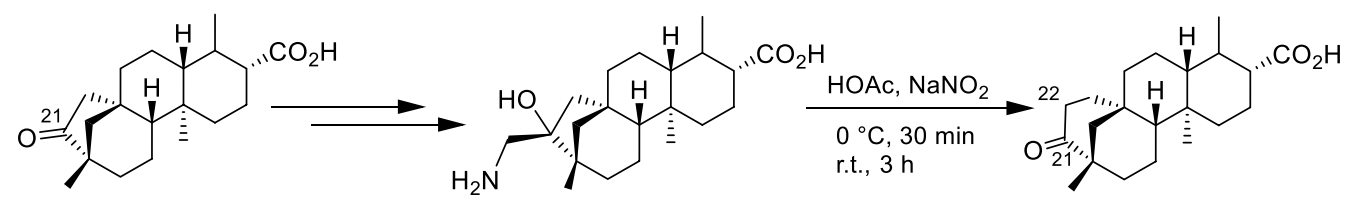

Scheme 17

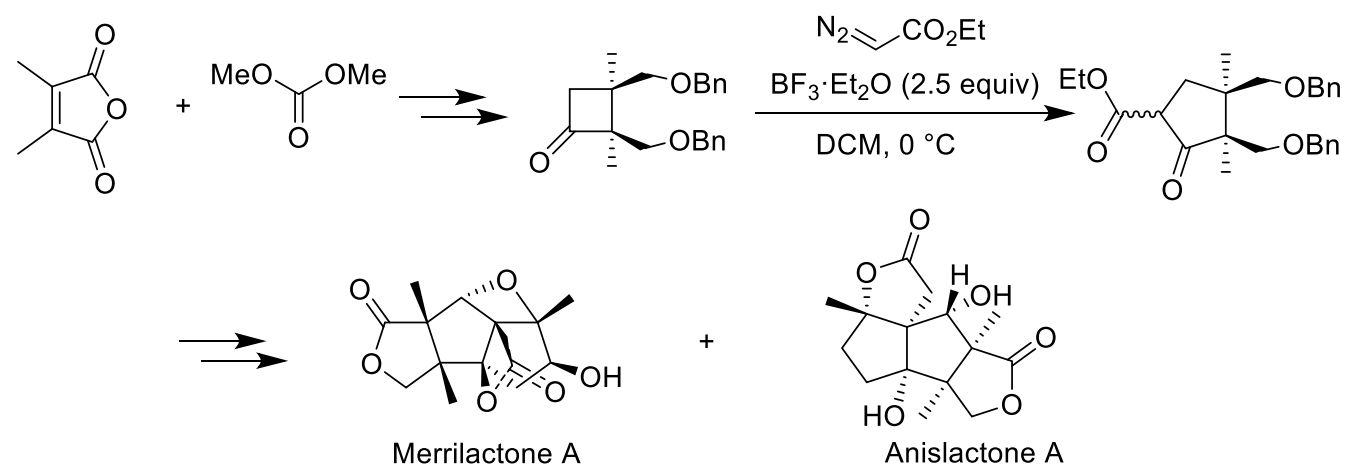




\section{Scheme 18}

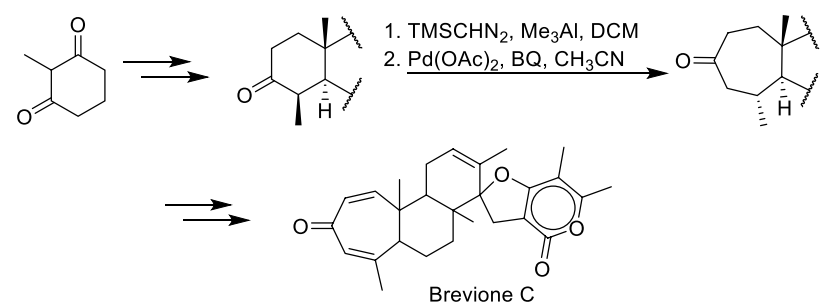

discovered working on phytotoxins, which could be used for the development of new herbicides. Therefore, it was also an interesting synthetic route of compounds as breviones grew. A ring expansion should make a cycloheptane ring

\section{Scheme 19}
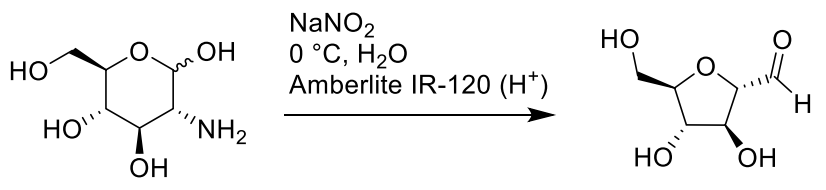

available for further synthesis. Using a TD-type intermediate together with a TMS derivative intermediate under different conditions in a one-pot experiment, cycloheptanones could be obtained from cyclohexanones (Scheme 18). The regioselectivity showed a dramatic change in the ratio of both regioisomers when performed in the dark and was first reported for this type of reactions. Macías et al. were the first that reported the influence of light in this kind of reactions. However, this new knowledge could lead to different possibilities since the most hindered side of a molecule is normally neglected during synthesis [56].

Plutschack et al. [57] applied the TD in a flow-based approach to contract a six-membered ring to a five-membered ring (Scheme 19). This was achieved using an acidic resin in which the educt, glucosamine hydrochloride, was passed over. The aim of this experiments was to synthesize larger quantities of a fluorescent fructose mimetic NBDM for uptake studies and further development of a Glut5-specific PET method, which could be used to monitor metabolic syndrome as well as cancer. The resin and the glucosamine were mixed with $\mathrm{NaNO}_{2}$ to produce nitrous acid in situ. Subsequently a basic resin was utilized for a required neutralization. The resulting solution was lyophilized overnight to isolate the compound. The conditions were optimized by focusing on the TD reaction using a catalytic amount of acid and sodium nitrite in water. The flow-based approach aided the use of high temperature and concentration and a throughput of $800 \mathrm{mg} / \mathrm{min}$ was achieved by the use of a $10 \mathrm{~cm}^{3}$ reactor at a flow rate of $5 \mathrm{~cm}^{3} / \mathrm{min}$ at $100{ }^{\circ} \mathrm{C}$. Comparing these results with the previously used batch production, a 64-fold increase in the throughput rate and a decrease in reaction time from 3 days to 1 day was observed. This modification of the TD helps to shift the paradigm of synthesis away from the one-pot-approaches to dynamic flow-approaches, which are not only easier to control, but can also lead to shorter reaction time due to higher throughput [57].

\section{Conclusions}

The TD rearrangement provides a simple and selective method for homologation process. It has several advantages compared to other homologation reactions, such as applicability over a wide range of different educts, retention of the configuration, high yield, any unwanted side reactions and high selectivity of the product. Due to these advantages, it provides an excellent tool in the synthesis of stereoselective complex molecules. In these cases, it may be employed for ring expansion but it is also a very valuable tool for ring contraction if needed. Due to these properties and the fact that in general the less substituted carbon is moving, the outcome of the reaction can be easily predicted beforehand.

Several improvements have been made in the last decades on the TD reaction, making this reaction even more powerful. One of these improvements is the use of LTMSD, which is originally a diazo compound, but the rearrangement proceeds in a TD-like way, combining the advantages of both reactions types, a one-step reaction not followed by formation of undesired products. For these reasons, nowadays the TD rearrangement can be easily applied in drug synthesis where high yields and stereoselectivity are of great importance.

Acknowledgements Open access funding provided by University of Vienna. We are grateful to the University of Vienna for financial support.

Open Access This article is distributed under the terms of the Creative Commons Attribution 4.0 International License (http://creativeco mmons.org/licenses/by/4.0/), which permits unrestricted use, distribution, and reproduction in any medium, provided you give appropriate credit to the original author(s) and the source, provide a link to the Creative Commons license, and indicate if changes were made.

\section{References}

1. King RB, Crabtree RH, Lukehart CM, Atwood D, Scott R (2006) Homologation reactions. Encyclopedia of inorganic chemistry. Wiley, Chichester

2. Pace V, Murgia I, Westermayer S, Langer T, Holzer W (2016) Chem Commun 52:7584

3. Miele M, Citarella A, Micale N, Holzer W, Pace V (2019) Org Lett 21:8261 
4. Miele M, D’Orsi R, Sridharan V, Holzer W, Pace V (2019) Chem Commun 55:12960

5. Ielo L, Touqeer S, Roller A, Langer T, Holzer W, Pace V (2019) Angew Chem Int Ed 58:2479

6. Monticelli S, Colella M, Pillari V, Tota A, Langer T, Holzer W, Degennaro L, Luisi R, Pace V (2019) Org Lett 21:584

7. Pace V, Holzer W, Ielo L, Shi C, Meng G, Hanna M, Szostak R, Szostak M (2019) Chem Commun 55:4423

8. Senatore R, Ielo L, Monticelli S, Castoldi L, Pace V (2019) Synthesis 51:2792

9. Karuppasamy M, Vachan BS, Vinoth P, Muthukrishnan I, Nagarajan S, Ielo L, Pace V, Banik S, Maheswari CU, Sridharan V (2019) Org Lett 21:5784

10. Castoldi L, Monticelli S, Senatore R, Ielo L, Pace V (2018) Chem Commun 54:6692

11. Senatore R, Castoldi L, Ielo L, Holzer W, Pace V (2018) Org Lett 20:2685

12. Touqeer S, Castoldi L, Langer T, Holzer W, Pace V (2018) Chem Commun 54:10112

13. Senatore R, Ielo L, Urban E, Holzer W, Pace V (2018) Eur J Org Chem 2018:2466

14. Castoldi L, Ielo L, Holzer W, Giester G, Roller A, Pace V (2018) J Org Chem 83:4336

15. Castoldi L, Ielo L, Hoyos P, Hernaiz MJ, De Luca L, An Alcantara, Holzer W, Pace V (2018) Tetrahedron 74:2211

16. Parisi G, Colella M, Monticelli S, Romanazzi G, Holzer W, Langer T, Degennaro L, Pace V, Luisi R (2017) J Am Chem Soc 139: 13648

17. Pace V, Castoldi L, Mazzeo E, Rui M, Langer T, Holzer W (2017) Angew Chem Int Ed 56:12677

18. Pace V, Pelosi A, Antermite D, Rosati O, Curini M, Holzer W (2016) Chem Commun 52:2639

19. Ielo L, Deri B, Germanò MP, Vittorio S, Mirabile S, Gitto R, Rapisarda A, Ronisvalle S, Floris S, Pazy Y, Fais A, Fishman A, De Luca L (2019) Eur J Med Chem 178:380

20. Ferro S, Deri B, Germanò MP, Gitto R, Ielo L, Buemi MR, Certo G, Vittorio S, Rapisarda A, Pazy Y, Fishman A, De Luca L (2018) J Med Chem 61:3908

21. Ferro S, De Luca L, Germanò MP, Buemi MR, Ielo L, Certo G, Kanteev M, Fishman A, Rapisarda A, Gitto R (2017) Eur J Med Chem 125:992

22. Li JJ (2009) Name reactions for homologations, Part I. Wiley, Chichester

23. Hesse M (1991) Ring enlargement in organic chemistry. VCH

24. Arndt F, Eistert B (1935) Ber Dtsch Chem Ges B 68:200

25. Wang Z (2010) Kowalski ester homologation. Comprehensive organic name reactions and reagents. Wiley, Hoboken

26. Gray D, Carmen Concellón A, Gallagher T (2004) J Org Chem 69:4849

27. Candeias NR, Paterna R, Gois PMP (2016) Chem Rev 116:2937

28. Reddy RE, Kowalski CJ (1993) Org Synth 71:146

29. Song Z-L, Fan C-A, Tu Y-Q (2011) Chem Rev 111:7523
30. Olson JA, Shea KM (2011) Acc Chem Res 44:311

31. Kürti L, Czako B (2005) Strategic applications of named reactions in organic synthesis. Academic Press, Cambridge

32. Reusch W (2016) Tiffeneau-Demjanov Rearrangement. In: Virtual textbook of OChem. Chemistry LibreTexts

33. Li JJ (2003) Name-Reactions. Springer, Berlin Heidelberg

34. Brocksom TJ, Brocksom U, de Sousa DP, Frederico D (2005) Tetrahedron Asymmetry 16:3628

35. Wovkulich PM (1991) Comprehensive organic synthesis. Elsevier, Amsterdam

36. Smith PAS, Baer DR (2011) The Demjanov and Tiffeneau-Demjanov ring expansions. Organic reactions. Wiley, Hoboken

37. Goldberg MW, Wydler E (1943) Helv Chim Acta 26:1142

38. Parham WE, Roosevelt CS (1972) J Org Chem 37:1975

39. Benjamin BM, Collins CJ (1961) J Am Chem Soc 83:3662

40. Collins CJ, Staum MM, Benjamin BM (1962) J Org Chem 27:3525

41. McClure M, Schiesser C, White J (2004) Aust J Chem 57:869

42. Chow L, McClure M, White J (2004) Org Biomol Chem 2:648

43. Liu H, Sun C, Lee N-K, Henry RF, Lee D (2012) Chem Eur J 18:11889

44. Wang Z (2010) Demjanov rearrangement. Comprehensive organic name reactions and reagents. Wiley, Hoboken

45. Cremlyn RJW, Garmaise DL, Shoppee CW (1953) J Chem Soc 1847

46. Coveney DJ (1991) The Semipinacol and Other Rearrangements. In: Trost BM, Fleming I (eds) Comprehensive Organic Synthesis, vol 3. Pergamon Press, Oxford, p 777

47. Lohoelter C, Schollmeyer D, Waldvogel SR (2012) Eur J Org Chem 2012:6364

48. Shi L, Meyer K, Greaney MF (2010) Angew Chem Int Ed 49:9250

49. Kouno I, Mori K, Kawano N, Sato S (1989) Tetrahedron Lett 30:7451

50. Huang J, Yokoyama R, Yang C, Fukuyama Y (2000) Tetrahedron Lett 41:6111

51. Birman VB, Danishefsky SJ (2002) J Am Chem Soc 124:2080

52. Inoue M, Sato T, Hirama M (2003) J Am Chem Soc 125:10772

53. Mehta G, Singh SR (2006) Angew Chem Int Ed 45:953

54. He W, Huang J, Sun X, Frontier AJ (2007) J Am Chem Soc 129:498

55. Hong B-C, Shr Y-J, Wu J-L, Gupta AK, Lin K-J (2002) Org Lett 4:2249

56. Macías FA, Carrera C, Chinchilla N, Fronczek FR, Galindo JCG (2010) Tetrahedron 66:4125

57. Plutschack MB, McQuade DT, Valenti G, Seeberger PH (2013) Beilstein J Org Chem 9:2022

Publisher's Note Springer Nature remains neutral with regard to jurisdictional claims in published maps and institutional affiliations. 